\title{
Temporary Grating Coupler Structures Using Localised Refractive Index Engineering
}

\author{
R. Topley ${ }^{1}$, G. Martinez-Jimenez ${ }^{1}$, L. O'Faolain ${ }^{2}$, N. Healy ${ }^{1}$, S. Mailis ${ }^{1}$, D. J. Thomson ${ }^{1}$, F. Y. \\ Gardes $^{1}$, A. C. Peacock ${ }^{1}$, D. N. R. Payne ${ }^{3}$, G. Z. Mashanovich ${ }^{1}$, G. T. Reed ${ }^{1}$ \\ 1. Optoelectronics Research Centre, University of Southampton, UK \\ 2. SUPA, School of Physics and Astronomy, University of St. Andrews, UK \\ 3. Nano Research Group, ECS, University of Southampton, UK \\ Contact Email: R.Topley@Soton.ac.uk
}

\begin{abstract}
We demonstrate an erasable grating coupler which allows optical device testing throughout the fabrication process without impairing final circuit performance. Refractive index variation is introduced using ion implantation and can be subsequently removed using laser annealing.

OCIS codes: (050.1950) Diffraction Gratings; (130.0130) Integrated Optics; (230.0230) Optical Devices; (050.0050) Diffraction and gratings.
\end{abstract}

\section{Introduction}

Unlike electronic integrated circuits, robust systems are not widely available for effectively autonomously testing optical integrated circuits at intermediate points on a waveguide, without introducing substantial losses into the devices. This makes quality control difficult as only the output of the entire optical circuit can be observed, providing information related to the complete optical circuit rather than localising a problem to the component level. A benefit of the Silicon on Insulator platform is its low cost; however processed silicon quickly becomes expensive. Each processing step costs money, and therefore testing is critical to reducing costs and increasing production yield.

Autonomous testing is essential for reducing the cost associated with optical integrated circuits (OIC) via an increased wafer yield. Autonomous testing after selected manufacturing steps, allows poor device performance or OIC failures to be detected early and to be either repaired using direct write method methods [1], or a cessation of further processing to reduce fabrication costs.

Autonomous testing methods for OIC have been considered previously. A metal grating coupler was fabricated directly onto a fibre facet in 2008 in order to create an optical test probe [2]. The probe was designed to interact with the evanescent field of the waveguide, and hence requires positioning in very close proximity to the waveguide surface for efficient coupling. Any large protruding features in close proximity to the waveguide such as electrodes or an oxide cladding will prevent comprehensive circuit testing with this optical probe. Furthermore the fibre based grating coupler was made using gold, which may contaminate samples preventing subsequent processing in CMOS fabrication facilities, though other work on metal gratings fabricated directly onto the waveguide surface suggests gold may not be critical to the process [3]. A CMOS compatible concept for wafer scale testing was presented later in 2011. The method used erasable Bragg grating reflectors $[4,5]$ to reflect light travelling in the waveguide, such that the light can then be collected by using a circulator and detector arrangement connected to the circuit input. This offers a wafer scale testing capability for devices spanning a small wavelength range, with typical Bragg gratings offering a few nanometres wavelength reflectivity. The other disadvantage of this method is that monitoring reflection may not facilitate testing of devices which have asymmetrical transmission characteristics, such as modulators.

Here we report a method for testing of optical circuits using erasable grating couplers with an aim to use the technology to improve wafer yield. Our method removes the issue of large residual test point losses. By utilising planar grating couplers formed using ion implantation for the measurement at intermediate points along the waveguide, and subsequently "erasing" them with a laser after testing is completed, resulting in a negligible residual loss. We have previously demonstrated implanted grating couplers with coupling losses of 5.5dB per coupler [6].

\section{Results \& Discussion}

A successfully erasable device will show both a substantial decrease in power output at the implanted grating test point, and an increase in power throughput. The implanted grating performance before and after laser annealing were measured. The results of these measurements are shown in Fig. 1. An implanted grating coupler with a period of $600 \mathrm{~nm}$ has been used for all of the results shown in this work. The uniform etched grating couplers used in this work 
have a coupling loss of $4.5 \mathrm{~dB}$ per coupler. The implanted couplers used in this work have a coupling loss of $6.6 \mathrm{~dB}$ per coupler, though implanted couplers have been demonstrated elsewhere operating at $5.5 \mathrm{~dB}$ per coupler [6]. All of the results have been normalised to a standard transmission measurement of the optical setup, which excludes the device under test.

A $488 \mathrm{~nm} \mathrm{CW}$ laser set to a power of $180 \mathrm{~mW}$ was used for annealing the grating. The sample was moved such that the laser passed over the implanted grating in a raster scan configuration, with an effective scan rate of $10 \mu \mathrm{m} / \mathrm{s}$. The laser spot size was measured to be $\sim 1.5 \mu \mathrm{m}$ with the separation between adjacent scans set to $0.5 \mu \mathrm{m}$, such that there was an overlap width of $\sim 1 \mu \mathrm{m}$ between successive scans. The large overlap ensures uniform melting of the surface by the Gaussian shaped beam. The laser scan was perpendicular to the waveguide and the path used for annealing extended $20 \mu \mathrm{m}$ beyond either side of the waveguide, the additional distance travelled removing any effects of acceleration in the sample movement, ensuring a constant exposure over the grating region undergoing the annealing process and also preventing excessive heating of the waveguide due to the increased dwell time encountered during the changes of direction in the raster pattern. In an optimized annealing process, the $20 \mu \mathrm{m}$ exposure extending beyond the grating coupler to be annealed could be reduced or potentially even removed by using a beam stop to block the laser beam during a change of direction or speed.

Fig. 1 shows a transmission measurement between an etched and implanted grating coupler, with a short $250 \mu \mathrm{m}$ single mode waveguide in between. After laser annealing the outcoupling performance of the implanted grating is attenuated by $21 \mathrm{~dB}$, as shown in Fig. 1, which demonstrates successful removal. The transmission through the erased grating section was also measured before and after annealing to ensure the grating has been effectively removed. Results showed a $5.7 \mathrm{~dB}$ increase in transmission through the grating region following laser annealing.

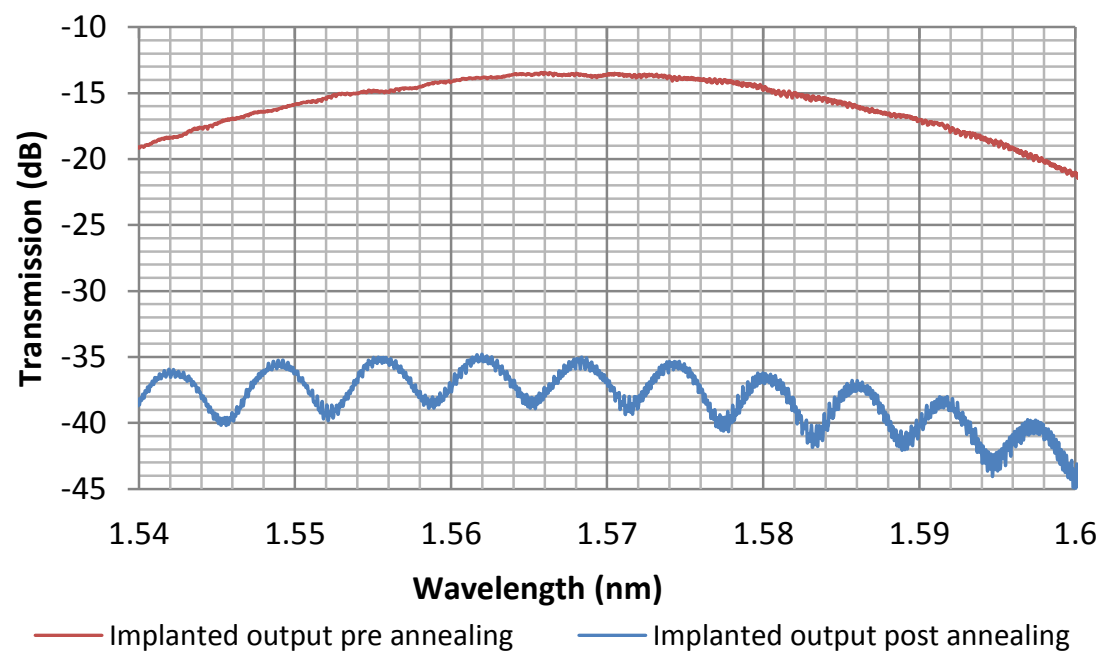

Fig. 1 - 100KeV Transmission loss for an etched to implanted grating structure before and after annealing

The data presented in Fig. 1 shows a clear reduction in the grating's coupling efficiency, though a low level of residual coupling is apparent, which suggests there is a small residual periodic refractive index change related to the implanted grating process. After annealing the implanted grating, it is possible that a residual loss will exist in the in the annealed region. The residual loss contribution has been calculated by measuring the loss of a control structure that has not been implanted, and subtracting this from the loss measured through a structure with an implanted grating that has been annealed. This indicates the residual loss introduced by implantation and subsequent annealing of an implanted grating structure is $\sim 0.3 \mathrm{~dB}$. A loss contribution of this small size may be observed from small fabrication variations within the surface relief grating couplers or a change in fibre alignment accuracy and hence requires further investigation with the annealing of cascaded devices to be conclusive.

Atomic force microscopy (AFM) was carried out to assess the effects of surface roughness and its potential contribution to a periodic refractive index change. Fig. 2 shows the AFM scan, from which a thin 30nm film of ZEP resist can be seen remaining on the surface, that has the same grating period and other characteristics as the original ZEP resist mask. Gratings formed from a periodic refractive index change upon the waveguide surface have been demonstrated previously, [3] with layer thicknesses as low as 5nm demonstrating coupling properties. Thus, the 
surface resist layer must be completely removed to avoid any residual coupling after testing, and to obtain optimal performance of the circuit.

ZEP has been shown to present a significantly increased etch resistance after exposure to high power electron beams, x-ray or heavy ion irradiation [7] due to cross-linking. The typically recommended process for removal of lithography resist after a standard solvent cleaning process is processing in an $\mathrm{O}_{2}$ plasma asher. An $\mathrm{O}_{2}$ plasma asher was performed prior to the AFM measurements for 10 minutes, at $200^{\circ} \mathrm{C}$ with a flow rate of $800 \mathrm{ml} / \mathrm{min}$ using an RF power of $800 \mathrm{~W}$. A longer $\mathrm{O}_{2}$ asher process may improve resist removal in future devices though a more robust solution is available by using a sacrificial thin film of silicon dioxide. $20 \mathrm{~nm}$ of silicon dioxide between the waveguide and the ZEP resist layers would be sufficient to buffer the waveguide surface from the harsh implant, and when the silicon dioxide layer is removed using a simple 20:1 buffered hydrofluoric acid etch, the resist would also be fully removed, leaving a much higher quality waveguide top surface.

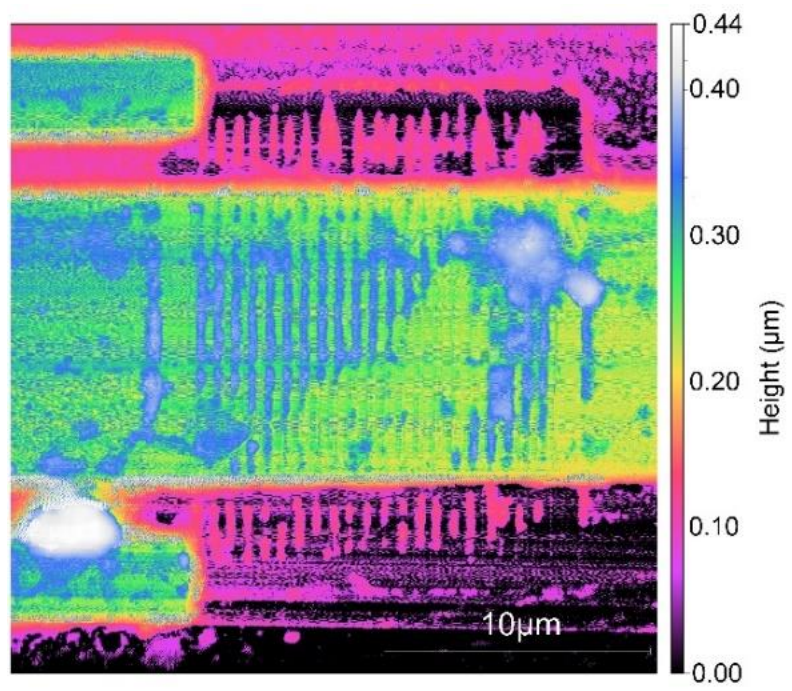

Fig. 2 - Surface profile of annealed implanted grating coupler. Original implant was germanium at $100 \mathrm{keV}, 1 \times 10^{15}$ ion $\mathrm{cm}^{-2}$

\section{Conclusion}

Grating couplers have been formed using ion implantation induced lattice disorder and have been removed by subsequent annealing. This novel system offers great promise as a wafer scale testing method which is durable and robust. The best implanted gratings have demonstrated an outcoupling loss of only $5.5 \mathrm{~dB}$, and results presented here show an attenuation of $21 \mathrm{~dB}$ following the removal of the grating via localised laser annealing.

Some residual coupling is shown and has been attributed to a thin film of resist which has been hardened by the annealing process. This issue can easily be overcome in future processing by utilising a thin layer of $\mathrm{SiO}_{2}$ underneath the implant mask, or by using a complete $\mathrm{SiO}_{2}$ hard mask in future designs. $\mathrm{SiO}_{2}$ has been tested with large dose implants before with no detriment to the etching process using buffered hydrofluoric acid.

\section{References}

[1] S. Howe, W. R. Headley, D. C. Cox, G. Z. Mashanovich, D. J. Thomson, and G. T. Reed, "Fabrication and tailoring of silicon photonic devices via focused ion beam," pp. 722011-722011, 2009.

[2] S. Scheerlinck, D. Taillaert, D. Van Thourhout, and R. Baets, "Flexible metal grating based optical fiber probe for photonic integrated circuits," Applied Physics Letters, vol. 92, pp. 031104-3, 2008.

[3] S. Scheerlinck, J. Schrauwen, F. Van Laere, D. Taillaert, D. Van Thourhout, and R. Baets, "Efficient, broadband and compact metal grating couplers for silicon-on-insulator waveguides," Optics Express, vol. 15, pp. 9625-9630, Jul 232007.

[4] R. Loiacono, G. T. Reed, G. Z. Mashanovich, R. M. Gwilliam, G. Lulli, R. Feldesh, et al., "Low-energy silicon-on-insulator ion implanted gratings for optical wafer scale testing," 2011, pp. 794310-794310-6.

[5] R. Loiacono, R. Topley, A. Nakyobe, G. Mashanovich, R. Gwilliam, G. Lulli, et al., "Very low energy implanted Bragg gratings in SOI for wafer scale testing applications," in Group IV Photonics (GFP), 2011 8th IEEE International Conference on, 2011, pp. 51-53.

[6] R. Topley, L. O'Faolain, D. J. Thomson, F. Y. Gardes, G. Z. Mashanovich, and G. T. Reed, "Planar surface implanted diffractive grating couplers in SOI," Optics Express, vol. 22, pp. 1077-1084, 2014/01/13 2014.

[7] T. Yamaguchi, K. Yamazaki, and H. Namatsu, "Influence of molecular weight of resist polymers on surface roughness and line-edge roughness," Journal of Vacuum Science \&amp; Technology B, vol. 22, pp. 2604-2610, 2004. 